THE CHEMICAL COMPOSITION OF THE WOLF-RAYET ENVELOPES

\title{
T. Nugis
}

Tartu Astrophysical Observatory, 202444, Tôravere, Estonia

ABSTRACT . Mode1-independent information on the chemical composition of WR stars is obtained from the lines of different ions arising at transitions between highly excited energy levels.

The chemical composition of WR stars strongly differs from the mean cosmic composition. Nuclear processed CNO products of $\mathrm{H}$-burning are revealed in WN envelopes and He-burning products in WC envelopes. The matter enriched with $\mathrm{He}$ and $\mathrm{N}$ is also present in some ring nebulae around WN stars.

\section{INTRODUCTION}

The Wolf-Rayet stars are in many respects complicated and interesting objects. So far, the physical background of intensive WR winds has remained a matter of some uncertainty. Furthermore, contemporary theoretical models have failed to fully explain the evolutionary status of WR stars. A specific property of WR spectra is the occurence of distinct sequences (WN, WC, WO). The different sequences of WR stars point to different chemical composition of observable layers. However some astronomers have attempted to prove that the occurence of different sequences is due to the different excitation and ionization conditions and does not reflect real differences in chemical composition.

Emission lines and violet-shifted P Cygni-type absorptions observed in WR spectra take their origin in the en riope. An envelope is defined as an expanding and continuously renewing outer region of the star which is formed by the more or less constantly blowing stellar wind. The inner limit of the envelope coincides with the radius of the stellar core which is defined as a region of formation of continuous stellar radiation and photospheric absorption spectrum (unshifted absorption lines). In WR spectra no definite photospheric absorption lines have been observed. These stars have very intensive mass outflow. The regions of winds with low expanding velocities $(\mathrm{v} \leq 100 \mathrm{~km} / \mathrm{s})$ are not seen at all because the optical depth of the layers above is much higher than unity for continuous absorption. In empirical modelling schemes the velocity law is not fixed a priori but has to be found by some subsequent iterative procedure. Presently, this seems to be a reasonable approach since the cause of intensive mass loss from WR stars remains unknown and the observed peculiarities of wind structure (coronal zones, deceleration zones, clouds etc.) are not fully understood. The empirical approach has 
been used in Nugis (1990a). Another type of WR models - standard WR models - have been suggested in the papers of Hamann and Schmutz (1987), Schmutz et al. (1989), Hillier (1987, 1989). In these models the velocity law is more or less strictly fixed.

So far, there are no fully satisfactory models available. Nevertheless, the existing models are able to explain some essential properties of WR spectra. Note that some parameters of WR stars are practically independent of the models applied and physical conditions assumed to exist in their envelopes. Here, chemical composition serves as an example of such parameters.

We shall first give the historical review of WR chemical composition determinations. Further, the derived compositions for different WR subtypes and ring nebulae around some WR stars are presented.

\section{DETERMINATION OF He/H RATIO}

Ambarzumian (1933) was the first to estimate quantitatively helium abundance in WR star envelopes. He analyzed the intensities of HeII $\lambda$ 4686(43) and HeII $\lambda 4860(8-4)+\mathrm{HI} \lambda 4861$ (4-2) lines. In concrete determinations a recombination theory was used. Ambarzumian found that helium compared to hydrogen is more abundant. This result has been confirmed by contemporary determinations. Although the recombination theory used is not a good approximation, the excitation conditions in WR envelopes of the studied subclasses are such that the line intensities are quite close to the recombination theory prediction.

Sobolev (1947) developed a powerful technique - escape probability method - for treating line formation in moving media.In earlier calculations of statistical equilibria equations for finding level populations and theoretical line intensities of $\mathrm{HI}$ and HeII, electron collisions and line emissions and absorptions induced by stellar continuous radiation were neglected. The $\mathrm{N}(\mathrm{He}) / \mathrm{N}(\mathrm{H})$ ratios were determined using such calculated HeII and HI line intensities by Sobolev (1952) and S1jusarev (1955). They confirmed the results of Ambarzumian (1933). After the appearance of the English version of Sobolev's paper (Sobolev, 1960) and of the modifications of that theory for spherically symmetric expanding envelopes considering the influence of line emissions and absorptions induced by continuous stellar radiation (Castor, 1970), new sets of calculations of $\mathrm{HI}$ and HeII statistical equilibrium equations were undertaken. In these studies electron collisions were taken into account (Castor and Van Blerkom,1970; Ilmas and Nugis, 1973). The calculations of theoretical HeI line intensities in the envelopes of WR stars based on the Castor modification of the Sobolev theory were first performed by Nugis (1973a) and 0egerle and 
Van Blerkom (1976). Although the assumed conditions in WR envelopes adopted in different studies are quite different, the theoretical intensity ratios for higher members of line series appear to be practically the same. These new studies gave also the overabundance of helium as compared to mean cosmic abundance (Castor and Van Blerkom, 1970; I1mas and Nugis, 1973). Rublev (1972) worked out the "limit decrement" method for deriving $\mathrm{N}(\mathrm{He}) / \mathrm{N}(\mathrm{H})$ ratios for $\mathrm{WR}$ envelopes. The essence of this method lies in the idea that for higher members of Pickering and Balmer series both the Menzel departure coefficients $b_{k}$ and Sobolev escape probability coefficients $\beta_{i k}$ tend to approach unity (for lower members of these series the coefficients $b_{k}, \beta$ ik are different from unity). From an observational point of view the highest members of Pickering series are difficult to apply due to the weakness of lines, blending problems and uncertainties of estimating the continuum level. In concrete calculations of statistical equilibria equations for level populations, the coefficients $b_{k}$ and $\beta_{i k}$ approach unity already at the intermediate values of $k(k \geq 9)$ for HeII. For practical purposes, e.g. for finding $\mathrm{N}(\mathrm{He}) / \mathrm{N}(\mathrm{H})$ ratios in WR envelopes it is the graphical method that is most convenient to apply. From the graphs we can first eliminate the lines which are blended with ions other than HeII and HI. Secondly, we can choose the region where $\beta_{i k}$ and $b_{k}$ have obtained already asymptotic regime $\left(b_{k} \beta_{i k}\right.$ $\approx 1$ ). Such an approach has been used consciously or intuitively by many astronomers (Rublev, 1972; Smith, 1973; Nugis, 1981, 1988, 1990c; Niemela, 1983; Smith and Willis, 1983; Conti et al.1983; Niedzielski, 1989). In some N(He)/ $\mathrm{N}(\mathrm{H})$ ratio determinations, unremoved blending may affect the results. Some lines used in concrete determinations ( $\lambda 4100$ (HeII 12-4), $\lambda 4200$ (HeII 11-4) and $\lambda 4339$ (HeII 10-4)) are blended by $N$ ions (WN stars) or by $C$ ions (WC stars). We have excluded these lines from the decrement study, However, some authors have included these lines, too, (Conti et al.,1983) and this accounts for the lower derived values for $\mathrm{N}(\mathrm{He}) / \mathrm{N}(\mathrm{H})$ ratio. Fig.1 contains the graphs of observed energies for Pickering series lines divided by the theoretical intensities corresponding to the case $b_{k} \beta_{i k}=$ 1. The difference between odd and even members of Pickering series gives us information about the presence of hydrogen. In determining $\mathrm{N}(\mathrm{He}) / \mathrm{N}(\mathrm{H})$ ratios it is essential to account for the ionization (stratification) effects, because hydrogen lines are formed in the whole envelope but HeII lines are formed only in certain parts of the envelope (in another part of the envelope HeI lines are formed). It is also possible that in some part of the envelope both HeII and HeI lines can be formed (ionization structure for different models may be different). When neglecting HeI contribution, only the lower limit for $\mathrm{N}(\mathrm{He}) / \mathrm{N}(\mathrm{H})$ ratio can be determined. 

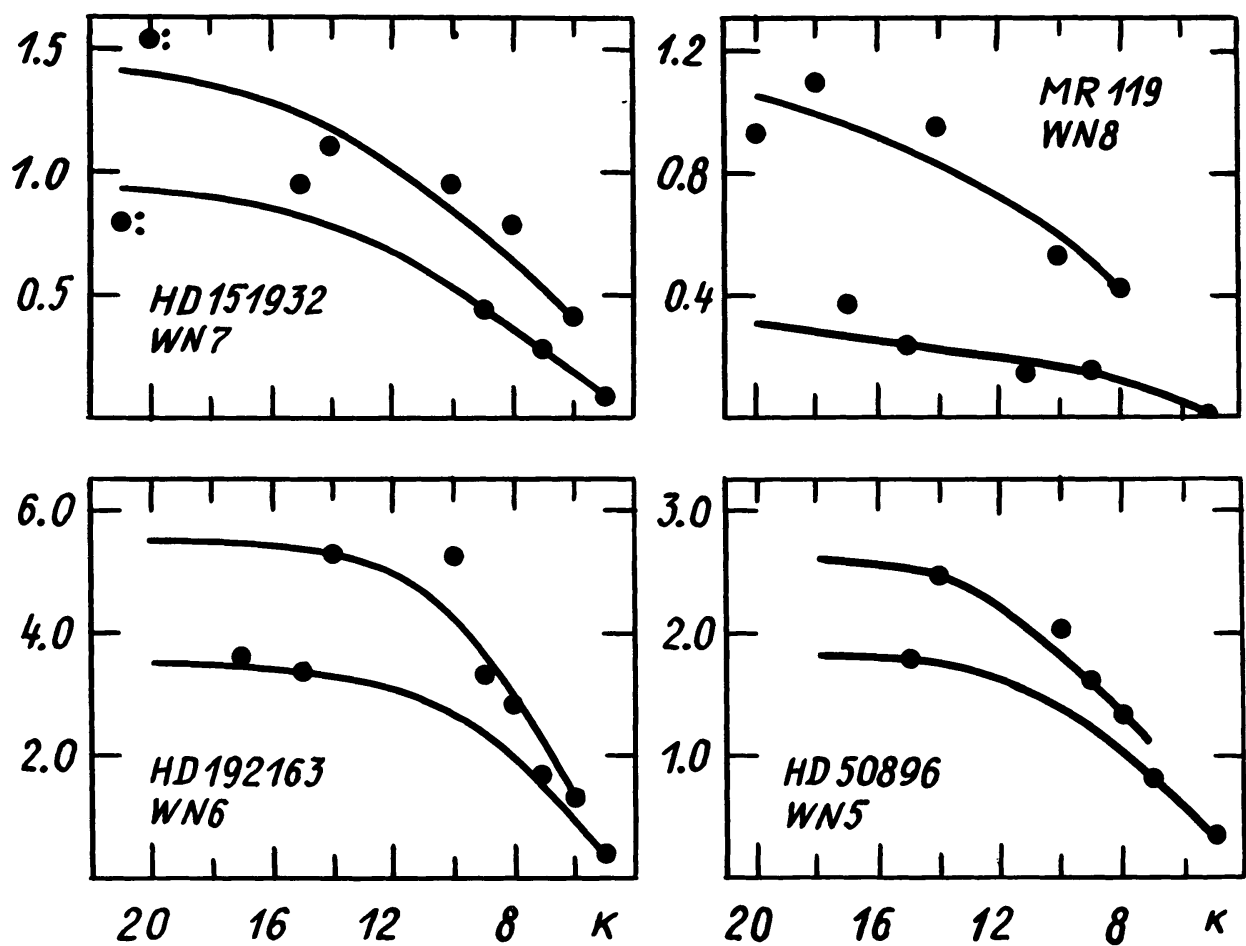

Fig. 1. The observed energies of Pickering series lines for WN stars divided by the theoretical intensities corresponding to the optically thin medium having LTE populations (๑). The scaled values are given $\left(10^{-16} \mathrm{E}_{\mathrm{k} 4} \mathrm{obs} / \mathrm{I}_{\mathrm{k} 4}\right) \cdot \mathrm{T}_{\mathrm{e}}=\mathrm{T}_{\star}$. The difference between odd and even members of Pickering series yields information about the presence of hydrogen. Observational data are derived from different sources (Nugis, 1989).

For some Galactic and LMC WN6-9 type stars Vreux et al. (1989) used near-IR lines of Paschen series and of HeIl k-6 series to derive the $\mathrm{N}(\mathrm{He}) / \mathrm{N}(\mathrm{H})$ ratio. From the higher members of these series they obtained that $\mathrm{N}(\mathrm{He}) / \mathrm{N}(\mathrm{H}) \approx 0.1-0.7$ for 8 wN6-9 stars. However, the contribution of HeI lines was excluded in their estimates. Therefore, their results ought to be regarded as the lower limit of $\mathrm{N}(\mathrm{He}) / \mathrm{N}(\mathrm{H})$ ratios for the studied stars. Possibly there exists some scatter of $\mathrm{N}(\mathrm{He}) / \mathrm{N}(\mathrm{H})$ ratios inside a subtype (Willis, 1982). As for the $\mathrm{N}(\mathrm{He}) / \mathrm{N}(\mathrm{H})$ ratio of WR stars in other galaxies there are few such studies available. No substantial differences exist compared to the Galactic WR stars (Willis, 1982; Smith and Willis, 
1983; Conti et al.,1983; Conti et al.,1989; Vreux et al.,1989). But this question needs further examination based on better observational material.

\section{THE DETERMINATION OF ABUNDANCES OF HEAVY ELEMENTS}

By heavy elements we mean elements heavier than helium. For heavy elements the first abundance estimates were made by Aller (1943) and by Zanstra and Weenen (1950). Aller used LTE populations together with the assumption that the envelope is optically thin at line frequencies and found that chemical composition of WR stars is near to normal. Aller's results were not confirmed by later studies. Zanstra and Weenen (1950) used recombination theory in predicting line intensities and estimated that for some WC stars the carbon abundance is very high, approximately one third of the number of helium atoms. Serious doubts were expressed as to the validity of this result. For the lines used the recombination theory is hardly a good approximation. Moreover, dielectronic recombination which was not considered in this study, ought to change the results considerably. But the mutual cancellation of different omitted processes makes their results quite close to the present estimates of carbon to helium ratio.

Based on intuitive considerations Gamov (1943) proposed excess carbon abundance in WC stars and excess nitrogen abundance in wN stars.

The first determinations of abundances for heavy elements by the use of the escape probability method and solutions of statistical equilibria for level populations where made by Castor and Nussbaumer, 1972 (CIII), Nugis 1973b (NV, CIV, NIV, NIII) and Nugis and Feklistova, 1973 (CIII). In these calculations electron collisions were taken into account but dielectronic recombinations were not considered. The latter processes ought to influence seriously the populations of some levels of CIII, NIV and NIII. Castor and Nussbaumer (1972) found that compared to the Sun in the WC8 star $y^{2}$ Vel the carbon abundance is at least 8 times higher. The ionization state of carbon was not determined in their study and the lower $1 \mathrm{i}-$ mit was obtained by assuming that carbon is predominantly three times ionized. Nugis (1973b) and Nugis and Feklistova (1973) obtained $\mathrm{N}(\mathrm{C}) / \mathrm{N}(\mathrm{He}) \approx$ $\approx 0.7-0.8$ for two WC stars and $\mathrm{N}(\mathrm{N}) / \mathrm{N}(\mathrm{He}) \approx 0.05-0.1$ for three wN stars. The results of latter studies are summarized in Nugis (1975). For $N$ ions in WN stars, the lines arising at transitions between quite low levels were included in determinations and for them the theoretical line intensities were underestimated due to the neglect of dielectronic recombination.

De Freitas Pacheco and Machado (1988) determined the $\mathrm{N}(\mathrm{C}) / \mathrm{N}(\mathrm{He})$ ratio for 8 WC stars using their solution of statistical equilibria equations 
for level populations of 4-level model of CIV atom. They obtained high $\mathrm{N}(\mathrm{C}) / \mathrm{N}(\mathrm{He})$ ratios increasing from early to late wC subtypes. This is an opposite trend compared with our trend and that suggested by Smith and Hummer (1988) and Torres (1988). The different result obtained by De Freitas Pacheco (1988) is due to the use of CIV $\lambda \lambda$ 5801, 5812 line, which are quite sensitive to concrete envelope modelling assumptions and especially to the assumed stellar radiation power in the unobservable EUV spectral region. For example, in our study we tried to use this line in estimating carbon abundances in WN stars.However, we had to neglect this idea because very different results were obtained depending on the adopted stellar radiation power in the CIV ground state continuum. We estimated upper limits to the of carbon abundance for some WN stars by using the line CIV $\lambda 5471$.

Associated with the appearance of UV satellites, new possibilities arose for chemical composition study. Willis and Wilson (1978) were the first to study $\mathrm{C}, \mathrm{N}$ abundances using the strongest UV lines (S2/68 experiment). The Sobolev escape probability method was applied when solving the equations of statistical equilibrium for level populations of the HeII, NIII-IV, CIII-IV ions. The one-effective-point-approximation was used to describe the whole envelope. The lines studied are sensitive to model assumptions. Their results ought to be regarded as underestimates. In the further UV studies the low-resolution IUE spectra for larger number of stars were investigated. Smith and Willis (1982) followed the same method as Willis and wilson (1978). Their $\mathrm{C} / \mathrm{He}$ and $\mathrm{N} / \mathrm{He}$ ratios must be regarded as the lower limits but $\mathrm{C} / \mathrm{N}$ ratios seem to be quite close to the real values.

The comparatively low $\mathrm{C} / \mathrm{He}$ ratio for wC stars in some studies seems to be caused by the use of lines dependent on concrete envelope models and assumed physical conditions. More realistic WC envelope models (Hillier (1989)) have yielded $\mathrm{N}(\mathrm{C}) / \mathrm{N}(\mathrm{He})=0.5$ for wC5 4-6 stars. Rather good agreement between high and low level subordinate lines was achieved at high carbon abundance also by the study of the author of this review by modelling some WR envelopes performed by the schemes described in Nugis (1989, 1990a, 1990c). High values for $\mathrm{C} / \mathrm{He}$ ratio in $\mathrm{WC}$ stars were obtained by using recombination theory also by Torres (1988).

New possibilities to derive quite reliable values for the abundances of some heavy elements arose when it became possible to observe the spectra in the IR region with sufficiently high resolution. In this region we can observe many lines of abundant ions arising at transitions between high energy levels (hydrogenic transitions) and also some forbidden lines of less abundant ions. For such lines even quite simple theore- 
tical calculation routines (recombination theory for hydrogenic lines and nebular approximation for forbidden lines) give the results which are close to those obtained within more elaborate modelling studies. Here one should also account for the nontransparency of the envelope in the continuum (Hummer et al. 1982). First the IR hydrogenic transitions of CIV, HeII and HeI were used to derive the lower limit for $\mathrm{C} / \mathrm{He}$ abundance ratio for $y^{2} \mathrm{Vel}(\mathrm{WC} 8)$ by Hummer et al (1982). Their investigation, based on the observational data obtained by Aitken et al. (1982). Aitken et al. (1982), derived lower limits for the abundance of $\mathrm{Ne}$ and $\mathrm{S}$ in the envelope of WC8 star of the binary $\gamma^{2}$ Vel from the forbidden lines of Ne and S. Van der Hucht and 01non (1985) obtained quite high value for Ne abundance $(\mathrm{N}(\mathrm{He}) / \mathrm{N}(\mathrm{He}) \approx 0.009)$ by using IRAS spectral data for $\gamma_{2} \mathrm{Vel}(\mathrm{WC})$. For the improved emission coefficients, the $\mathrm{N}(\mathrm{Ne}) / \mathrm{N}(\mathrm{He})$ ratio appears to be $\approx 0.001$ (Barlow et a1., 1988). Smith and Hummer (1988) used hydrogenic type transitions in the IR spectral range to derive $\mathrm{N}(\mathrm{C}) / \mathrm{N}(\mathrm{He})$ ratios for 17 southern WC stars. They used the spectra in the $\mathrm{H}$ and $\mathrm{K}$ bands obtained with the Infrared Photometer/Spectrometer on the 3.9-m Anglo-Australian Telescope. The recombination theory was used to predict theoretical line intensities including collisional redistribution. They found that carbon abundance in WC star envelopes is high and that carbon abundance increases when going from later WC subtypes to earlier subtypes.

\section{DERIVED CHEMICAL COMPOSITION IN WR ENVELOPES AND IN THE RING NEBULAE AROUND THEM}

From the previous sections we can conclude that chemical composition of WR envelopes seems to be strongly abnormal. But some astronomers have still tried to prove that the chemical composition of WR stars is quite normal. In this aspect the studies of Bhatia and Underhill $(1986,1988)$ serve as an example. Their parameters are quite different from the usually adopted values. Therefore we face the problem of the model-dependence of different estimations of chemical composition. By solving statistical equilibrium equations for level populations Bhatia and Underhill (1986) found that compared with helium, hydrogen is more abundant in WR envelopes. In concrete determinations they used the HeII $\lambda 4542(9-4)$, HeII $\lambda 4859(8-4)+$ HI $\lambda 4861(4-2)$ and HeII $\lambda 5412(7-4)$ lines. For these lines their models predict $b_{k} \beta_{i k}>1$. But the graphs presented (Fig. 1) give $b_{k} \beta_{i k}<1$ for Pickering lines with $k \leq 9$ for every WR star. In constructing our graphs we used the value $\mathrm{T}_{e}=\mathrm{T}_{*}$ whereas $\mathrm{T}_{*}$ was taken from our estimates (Nugis, 1990b). The use of $\mathrm{T}_{\mathrm{e}}=100000 \mathrm{~K}$, as adopted by Bhatia and Underhill (1986) does not change the qualitative shape of the graph. Therefore the models of Bhatia and Underhill predict a wrong run of Pic- 
kering series decrement and their normal ratio of $\mathrm{N}(\mathrm{H}) / \mathrm{N}(\mathrm{He})$ for $W R$ stars is due to the use of inadequate models together with model-depending lines in concrete estimations. Even their models give for higher Pickering and Balmer series lines the same results about $b_{k} \beta_{i k} \rightarrow 1$. Thus, we conclude that helium is strongly overabundant in WR star envelopes. From our investigation it can be concluded that the line intensities predicted by models of Bhatia and Underhill $(1986,1988)$ are in serious conflict with the observed line spectra, but the line intensity ratios of subordinate lines arising at transitions between high energy levels are quite close to the ratios predicted by our WR wind models. However, note that reliable results can be obtained by inadequate models too, provided, that "right" lines are used. For subordinate lines arising at transitions between high energy levels even very simple calculation schemes (reconbination theory) predict quite close results to those obtained by more realistic envelope models and appropriate statistical equilibrium calculations for level populations.

Table 1 gives the most recent determinations of chemical compositions for different subtypes of WR stars. For wo stars Kingsburgh and Barlow (1990) have determined from high-n recombination lines that these stars have nearly the same $\mathrm{N}(\mathrm{C}) / \mathrm{N}(\mathrm{He})$ ratios as early wC stars and that $\mathrm{N}(0) / \mathrm{N}(\mathrm{H}-$ e) ratios may be somewhat higher than those for WC stars. According to our estimates, the values in Table 1 ought to be regarded as close to the real values with an accuracy of factor 2-3. Carbon abundance is increasing from later WC classes to earlier classes. In the case of WN classes some chemical evolution from later classes to earlier classes seems to be present as well. The chemical composition of WR stars strongly differs from the mean cosmic composition. Nuclear-processed CNO products of $\mathrm{H}-$ burning are revealed in the WN envelopes and He-burning products in the WC envelopes.

In 1965 a new type of emitting nebulae - the ring nebulae around WR stars - were discovered (Johnson and Hogg, 1965). Johnson and Hogg explained them as wind-blown cavities in the nearby interstellar matter. At present ring nebulae have been detected around more than twenty WR stars in Galaxy and LMC. Such nebulae exist mainly around wN stars, but some of them are detected also around WC stars. According to the present understanding some of them indeed can be formed by the interaction of stellar wind with IS matter, but some of them may be formed by ejection of some part the of matter by the central star. In morphological studies, four types of ring nebulae have been distinguished (Chu, 1981).

The chemical composition studies of ring nebulae are very important in contributing to our understanding of the of ring nebulae formation. 
Furthermore, it contributes to a deeper understanding of the properties of central WR stars. The abundance studies for these objects are mostly based on observations of the brightest part of the nebulae or on the average spectra. Such an approach may sometimes smooth out important information. Some abundance analysis of spatially revolved observations of different parts of the ring nebula NGC 6888 surrounding the WN6 star HD 192163 are presented by Kwitter (1981). For the nebulae NGC 2359 and RCW 78 surrounding the wN5 star HD 56925 and the WN8 star HD 117688, the spatially resolved spectroscopic study of possible abundance inhomogeneities is reported by Esteban et al. (1990). Forbidden lines of heavy elements and recombination lines of helium and hydrogen allow to estimate quite correctly the abundances in the nebula. In four ring nebulae surrounding WN stars quite substantially enhanced abundances of $\mathrm{N}$ and $\mathrm{He}$ as compared to normal (differences up to 3-10 times) have been detected. These are NGC 6888 (Kwitter, 1981), S308, RCW 58 (Kwitter, 1984) and RCW 78 (Esteban et al. 1990). In some others only very small enrichment may be present - anonymous nebula around HD 191765 and NGC 3199 (Kwitter, 1984) and NGC 2359 (Esteban et al. 1990).

It is easy to explain the observed chemical enrichments as being due to the addition to the normal composition ISM some amount of enriched by $\mathrm{He}$ and $\mathrm{N}$ matter of the WN winds (or ejecta).

It would be very interesting to carry out a composition study of ring nebulae surrounding WC stars. This ought to give essential information on the evolutionary status of these stars. Present theoretical evolutionary schemes cannot yet fully explain the observed properties of these stars.

\section{CONCLUDING REMARKS}

The chemical composition of WR stars strongly differs from the mean cosmic composition. In the case of WN stars nuclear-processed CNO products of H-burning are revealed in their envelopes and the weakly enriched matter is also present in some ring nebulae around them.In the case of WC and wO stars the products of He-burning are revealed in their envelopes.

Carbon abundance is increasing from later to earlier wC subtypes. In the case of WN classes some chemical evolution from later to earlier subtypes seems to be present as well.

The oxygen abundance derived for WC stars differs from the prediction of the calculations of WR evolution (Prantzos et al., 1986; Langer and E1 Eid, 1986; Maeder, 1987). The discrepancy with evolutionary calculations 


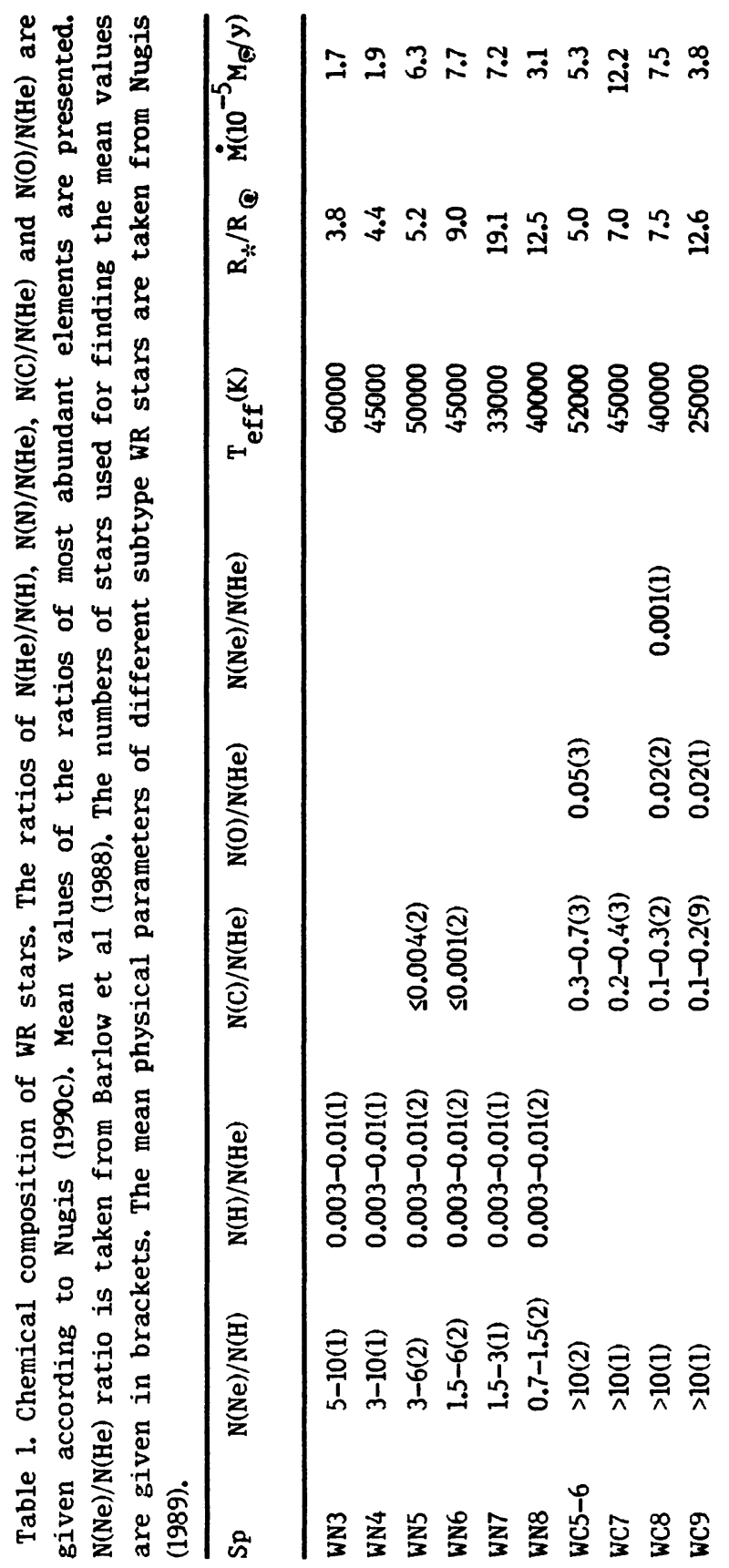


was found also in the case of Ne abundance in the envelope of WC8 component of binary $\gamma^{2} \mathrm{Vel}$ (Barlow et al., 1988).

In most cases WN stars do not seem to evolve into WC stars. This follows from the fact that mass loss rates of the earliest wN stars are lower than those for later types (probably the end points of WN sequence evolution are hot helium-rich stars) and from the fact that the radii of latest WC stars are much higher than for early WN stars. Some stars possibly reach the WR phase as WC stars. After the WC phase there ought to follow the wo phase and these stars may end as supernovae as predicted by the evolutionary theory.

\section{References}

Aitken, D.K., Roche, P.E. and Allen, D.A., 1982. M.N.R.A.S. 200, 69P. Aller, L.H., 1943. Ap. J. 97, 135.

Ambarzumian, V., 1933. Pulkovo Obs. Circ. No 7, 11.

Barlow, M.J., Roche, P.E. and Aitken, D.K., 1988. M.N.R.A.S. 232, 821.

Bhatia, A.K. and Underhil1, A.B., 1986. Ap. J. Supp1. $\underline{60}, 232$.

Bhatia, A.K. and Underhill, A.B., 1988. Ap. J. Supp1. 67, 187.

Castor, J.I., 1970. M.N.R.A.S. 149, 111.

Castor, J.I. and Nussbaumer, H., 1972. M.N.R.A.S. 155, 293.

Castor, J.I. and Van Blerkom D., 1970. Ap. J. 161, 485.

Chu, Y.-H., 1981. Ap. J. 249, 197.

Conti, P.S., Leep, E.M. and Perry, D.N., 1983. Ap. J. 268, 228.

Conti, P.S., Massey, P. and Garmany, C.D., 1989. Ap. J. 341, 113.

de Freitas Pacheco, J.A. and Machado, M.A., 1988. A.J. 96, 365.

Esteban, C., Vilchez, J.M., Machado, A. and Edmunds, M.G., 1990. Astron. Astrophys. $227,515$.

Gamov, G., 1943. Ap. J. 98, 500.

Hamann, W.-R. and Schmutz, W., 1987. Astron. Astrophys. 174, 173.

Hillier, D.J., 1987. Ap.J. Suppl. 63, 947.

Hillier, D.J., 1989. Ap.J. $\underline{347} 392$.

Hummer, D.G., Barlow, M.J. and Storey P.J., 1982. In: "Wolf-Rayet Stars: Observations, Physics, Evolution", Proc. IAU Symp. No 99, eds. C. de Loore, A.J. Willis, Dordrecht, Reidel, p. 79.

Ilmas, M. and Nugis, T., 1973. Tartu Astrofiuis. Obs. Teated No 44, 19 (in Russian).

Johnson, H.M. and Hogg, D.E., 1965. Ap.J. 142, 1033.

Kingsburgh, R.L. and Barlow, M.J. 1990. Proc. IAU Symp. No 143 (in press).

Kwitter, K.B., 1981. Ap. J. 245, 154.

Kwitter, K.B., 1984. Ap. J. $287,840$.

Langer, N. and E1 Eid, M.E., 1986. Astron. Astrophys. 167, 265. 
Maeder, A., 1987. Astron. Astrophys. 173, 247.

Niedzielski, A., 1989. Acta Astronomica 39, 251.

Niemela, V.S., 1983. In:"Wolf-Rayet Stars: Progenitors of Supernovae?", eds. M.C. Lortet, A. Pitault, Paris, l'observatoire, p.III.3.

Nugis, T., 1973a. Tartu Astrofuiius. Obs. Teated No 44, 53 (in Russian).

Nugis, T., 1973b. Tartu Astrofuiuis. Obs. Teated No 44, 83 (in Russian).

Nugis, T., 1975. In: "Variable Stars and Stellar Evolution". Proc. IAU No

67, eds. V.E. Sherwood, L. Plaut, Dordrecht, Reidel, p. 291.

Nugis, T., 1981. Tartu Astrofuius. Obs. Pub1. 48, 109.

Nugis, T., 1988. Tartu Astrofuiuis. Obs. Teated No 89, 89 (in Russian).

Nugis, T., 1989. Tartu Astrofuius. Obs. Teated No 94, 3.

Nugis, T., 1990a. Astrofiz. 32, 85 (in Russian).

Nugis, T., 1990b. Tartu Astrofuiuis. Obs. Publ. 53 (in press)

Nugis, T., 1990c. Proc. IAU Symp. No 143 (in press).

Nugis, T. and Feklistova, T., 1973. Tartu Astrofuius. Obs. Teated No 44, 145 (in Russian).

Oegerle, W.R. and Van Blerkom D., 1976. Ap. J. 206, 150.

Prantzos, N., Doom, C., Arnould, M. and de Loore, C., 1986. Ap. J. 304, 695.

Rublev, S.V., 1972. Izv. Spets. Astrofiz. Obs. 4 , 3 (in Russian).

Schmutz W., Hamann W.-R. and Wessolowski U., 1989. Astron. Astrophys. 210 , 236.

Sljusarev, S.G., 1955. Astron. Zh. 32, 346 (in Russian).

Smith, L.F., 1973. In: "Wolf-Rayet and High-Temperature Stars". Proc. IAU Symp. No 49, eds. M.K.V. Bappu, J. Sahade, Dordrecht, Reidel, p.15.

Smith, L.F. and Hummer D.G., 1988. M.N.R.A.S. 230, 511.

Smith, L.J. and Willis, A.J., 1982. M.N.R.A.S. 201, 451.

Smith, L.J., and Willis, A.J., 1983. Astron. Astrophys. Supp1. 54, 229.

Sobolev, V.v. 1947. "The Moving Envelopes of Stars", Leningrad State University (in Russian)

Sobolev, V.V., 1952. In:"Theoretical Astrophysics", ed. V.A. Ambarzumian, Gostehizdat, Moscow, p. 479 (in Russian).

Sobolev, V.V., 1960. "The Moving Envelopes of Stars", Harvard Univ. Press.

Torres, A.V., 1988. Ap. J. $\underline{325}, 759$.

Van der Hucht, K.A. and 01non, F.N., 1985. Astron. Astrophys. 149, L17.

Vreux, J.M., Dennefeld, M., Andrillat, Y. and Rochowicz K., 1989. Astron. Astrophys. Supp1. 81, 353.

Willis, A.J., 1982. In:"Wolf-Rayet Stars: Observations Physics, Evolution", Proc. IAU Symp. No 99, eds. C.de Loore, A.J. Willis, Dordrecht, Reidel, p. 87.

Willis, A.J. and Wilson, R., 1978. M.N.R.A.S. 182, 559.

Zanstra, H. and Weenen J., 1950. Bull. Astron. Inst. Neth. 11, 165. 